\title{
Nitrogen application and intercropping advantageously promote microbial community diversity and physio-chemical characteristics in mulberry and alfalfa rhizosphere soil
}

\author{
Xiuli Zhang ${ }^{1}$, Zhiyuan Teng ${ }^{1}$, Huihui Zhang ${ }^{2}$, Dunjiang Cai ${ }^{3}$, Jingyun Zhang ${ }^{4}$, Guangyu \\ Sun $^{1}$, and Fanjuan Meng ${ }^{1}$ \\ ${ }^{1}$ Northeast Forestry University \\ ${ }^{2}$ Northeast Agricultural University \\ ${ }^{3}$ Institute of Crop Development \\ ${ }^{4}$ Heilongjiang Academy of Land Reclamation and Agricultural Sciences
}

May 5, 2020

\begin{abstract}
Mulberry intercropped with alfalfa is a popular agroforestry system in China, which can produce high forage yields with high protein. To investigate the advantages of intercropping as well as the responses of mulberry-alfalfa intercropping systems to nitrogen application, we studied changes in the soil microbial communities and physiochemical properties in the rhizosphere of intercropped mulberry and alfalfa under nitrogen application. Nitrogen application increased available nitrogen contents (AN) and activities of urease (SUR) in rhizosphere soil of mulberry and alfalfa, but reduced soil organic canmatter (OM), irrespective of the cropping system (monoculture or intercropping). Nitrogen application increased soil $\mathrm{pH}$ in the rhizosphere of mulberry and alfalfa in monoculture, but reduced soil $\mathrm{pH}$ in the intercropped system. Nitrogen application and intercropping enhanced the soil water content (SWC) in mulberry rhizosphere soil and decreased SWC in alfalfa rhizosphere soil. We observed significant differences in the microbial communities inhabiting mulberry and alfalfa rhizosphere soils, indicating that mulberry and alfalfa are strategically complementary in terms of carbon sources. The Shannon-Weaver (H'), Simpson index (D), and McIntosh diversity (U) values were higher in rhizosphere soil of intercropped mulberry compared with mulberry in monoculture without nitrogen supply. There were no significant differences between mulberry in monoculture with nitrogen application (MNE) and mulberry intercropped alfalfa without nitrogen (M0). The results of the principal components analysis showed that MNE and M0 clustered in the scattered plots. We found no significant differences for H' and D between monoculture and intercropping alfalfa systems, irrespective of the nitrogen application. Nitrogen application, intercropping, and intercropping with nitrogen increased the numbers of carbon sources, and relative use rates exceeded $4 \%$. Nitrogen application and intercropping reduced the numbers of available carbon sources in alfalfa. Redundancy discriminatory analysis results suggest that $\mathrm{pH}$ and $\mathrm{SWC}$ were positively related with mulberry treatments and negatively with the alfalfa treatments.
\end{abstract}

\section{Introduction}

Legumes are important components of intercropping systems and fix atmospheric nitrogen in root nodules, which can be used by associated crops (Lipman, 1912). Alfalfa (Medicago sativa L.) is a widely distributed forage legume and rich in protein (Baslam, Antolín, Gogorcena, Muñoz, \& Goicoechea, 2014), it has become one of main forage sources for animal husbandry. Intercropped with alfalfa could increase microbial activity and diversity in the rhizosphere of Siberian wild rye (Elymus sibiricus L.) (Y. M. Sun et al.) and Mulberry (Morus alba L.) (Zhang, Wang, Hu, \& Sun). Mulberry, a forage deciduous tree, has been recognized as a suitable alternative for supplementing live-stock diets (Sanchez, 1999; Wang, Yang, Bo, Ding, \& Cao, 2012) in sustainable animal production systems (e.g. agro-forestry or agro-silvopastoral systems), with a higher 
biomass amount than that of most traditional forages (Sanchez, 1999). In terms of digestible nutrients, mulberry leaves and young stems are highly palatable and digestible (70-90\%) for herbivorous animals and can also be fed to monogastrics. Alfalfa intercropped with mulberry is a new agroforestry system in China and has become an important and economic feasible planting pattern (Zhang et al., 2018). The two plants are both perennial forages with high protein contents; they are mowed two to three times a year and remove large amounts of soil nitrogen. Nitrogen supply is a determining factor in agricultural systems and affects biomass quantity and quality of alfalfa and mulberry. It has been reported that the percentage of $\mathrm{N}$ obtained through alfalfa $\mathrm{N}_{2}$ fixation in a pure stand is only 33 to $80 \%$ (Heichel \& Vance, 1979). Moreover, alfalfa mainly relies on the uptake of nitrogen from the soil to meet its $\mathrm{N}$ demands during the seedling stage (not forming nodules) or after mowing (low photosynthetic capacity) (Teuber, Levin, Sweeney, \& Phillips, 1984). The promoting effect of nitrogen application on alfalfa yield is obvious in the first planting year. Microbial communities are considered the drivers of soil functions and are involved in organic matter formation and decomposition (Condron, Stark, O'Callaghan, Clinton, \& Huang, 2010), respiration (Liu et al., 2018), nutrient mineralization and cycling as well as water infiltration (Chu \& Grogan, 2010). Any decrease in microbial diversity or abundance may adversely affect nutrient absorption from the soil (Giller, Witter, \& Mcgrath, 1998), while in turn, the structure and diversity of the soil microbial communities are affected by vegetation composition and diversity (Ladygina \& Hedlund, 2010), and in continuous cultivation systems, microbial communities show a reduced diversity (Jiao, 2006). Therefore, intercropping is an important approach to increase microbial diversity and quantity (Tang et al., 2014), resulting in increased productivity (Mao et al., 2012).

At present, the effects of nitrogen application and intercropping on the soil microbial diversity of the mulberry and alfalfa rhizosphere in the first planting year are not clear. The Biolog EcoPlate contains three replicate wells of 31 of the most useful carbon sources for soil community-level physiological profiling (CLPP) of heterotrophic bacterial assemblages capable of being metabolically active and growing in plates (Stefanowicz, 2006). This method is based on the fact that different microorganisms use different carbon sources, which enables the determination of the functional diversity of a microbial community (Amador \& Görres, 2007). In comparative studies using CLPP and high-throughput sequencing (16S rRNA and ITS rRNA) (Huang et al., 2017), similar results were found in terms of the effect of land-use change on the soil microbial community structure (Dong, Yao, De-Yong, \& Huang, 2008); however, the advantage of the Biolog approach is that it can be performed simply and conveniently and does not require substantial costs and resources (Classen, Boyle, Haskins, Overby, \& Hart, 2003; Jay L. Garland, 1997).

In this study, we used the Biolog Ecoplate ${ }^{\mathrm{TM}}$ microplate technique to assess the effects of nitrogen and intercropping on the microbial community structure and function of mulberry and alfalfa rhizosphere soil. In addition, we analyzed the relationship between the diversity of mulberry and alfalfa rhizosphere microbes (in terms of carbon source use) and the soil physiochemical properties. We anticipate that our findings will provide a reference for the adequate cultivation and management of agroforestry systems of mulberry intercropped with alfalfa.

\section{Material and methods}

\subsection{Field experiment}

The experiment was performed at the Crop Institute (Jiamusi) of the Heilongjiang Academy of Land Reclamation Science (latitude, $46^{\circ} 17.02$ ' N; longitude, $46^{\circ} 53.16$ 'E, northeast China). Mean elevation is 81.2 $\mathrm{m}$ above sea level, average annual precipitation is $460 \mathrm{~mm}$, and nearly $59 \%$ of the total rainfall is received during the northwest monsoon from July to September. The active accumulated temperature ([?]10degC) is 2,600degC per year, with a frost-free period of $137 \mathrm{~d}$. The soil is classified as meadow soil, with the following properties: $\mathrm{pH} 6.5$, available nitrogen $89.2 \mathrm{mg} \mathrm{kg}^{-1}$, available phosphorus $128.2 \mathrm{mg} \mathrm{kg}^{-1}$, available potassium $106 \mathrm{mg} \mathrm{kg}{ }^{-1}$. Mulberry (Morus alba L.c v. qiuyu ) seedlings with high yield, cold- and drought-resistant, were provided by the Heilongjiang Institute of Silkworm breeding; alfalfa (Medicago sativa L.c v. zhaodong ) seeds with a strong wintering ability and a high yield were obtained from Institute of Crop development the Heilongjiang Academy of Land Reclamation Science. 


\subsection{Experimental design}

The experimental design consisted of a split plot experiment with two factors (monoculture and intercropping); the treatments included nitrogen application and no nitrogen application, with a total area of about $1,400 \mathrm{~m}^{2}$. Each intercropping treatment covered an area of $60 \mathrm{~m}^{2}(5 \mathrm{mx} 12 \mathrm{~m})$. The configured lines of intercropped mulberry and alfalfa were two and four, respectively. The planting distance of mulberry was $45 \mathrm{~cm}$, with a row spacing of $60 \mathrm{~cm}$ (Figure 1). For alfalfa, row spacing was $30 \mathrm{~cm}$. To avoid nitrogen leaching and seedling burn, the nitrogen was applied at the bud stage of alfalfa through an opening ditch. Phosphorus, potassium, and other essential elements were applied in mid-April. In particular, we used 23 $\mathrm{kg}^{*} 667 \mathrm{~m}^{-2}$ of superphosphate and $6.67 \mathrm{~kg}^{*} 667 \mathrm{~m}^{-2}$ of potassium chloride. To avoid interference by other elements, nitrogen was applied as urea $(46 \% \mathrm{~N})$. In both systems (monoculture and intercropping), the same amounts of nitrogen were applied. For alfalfa, we applied $5.86 \mathrm{~kg}^{*} 667 \mathrm{~m}^{-2}$ of urea, while for mulberry, 22 $\mathrm{kg}^{*} 667 \mathrm{~m}^{-2}$ were applied.

\subsection{Soil sampling}

On 4 July 2016, at the mid-bloom stage of alfalfa in the first planting year, soil samples were collected from mulberry and alfalfa rhizosphere soils by shaking the soil off the root. One part of the sample was used for soil analyses, while the other part was used for the determination of carbon source use via Biolog plates. For extraction, $10 \mathrm{~g}$ of soil were shaken with $100 \mathrm{~mL} 0.1 \mathrm{M}$ Tris buffer (pH 7.5) for $10 \mathrm{~min}$ and centrifuged for $10 \mathrm{~min}$ at $2,600 \mathrm{~g}$. The supernatant was diluted, and $150 \mathrm{~mL}$ of the $10^{-3}$ dilutions were inoculated into Biolog-Ecoplates (Biolog, Hayward, CA, USA). Each of the 96-well plates contained 31 different carbon sources separately and a blank well, each replicated three times. The plates were incubated at $28 \mathrm{degC}$, and substrate use condition was monitored by measuring absorbance via an automated plate reader (ELX808, Lab systems, Helsinki, Finland) at $590 \mathrm{~nm}$ every $24 \mathrm{~h}$ for $192 \mathrm{~h}$.

\subsection{Calculation of metabolic functional diversity indices}

Average well-color development (AWCD) was calculated by averaging the final absorbance values for all substrate wells, as described by Garland (Jay L. Garland, 1997):

$\mathrm{AWCD}=[?]\left(\mathrm{C}_{\mathrm{i}}-\mathrm{R}\right) / 31$,

where $\mathrm{Ci}$ is the absorbance value of each reaction well at different incubation times at $590 \mathrm{~nm}$, excluding the control well, and $\mathrm{R}$ is the absorbance value of the control well. The Shannon index $(\mathrm{H})$ was calculated as follows:

$\mathrm{H}=-[?]\left(\mathrm{P}_{\mathrm{i}} \mathrm{x} \ln \mathrm{P}_{\mathrm{i}}\right), \mathrm{P}_{\mathrm{i}}=\left(\mathrm{C}_{\mathrm{i}}-\mathrm{R}\right) /[?]\left(\mathrm{C}_{\mathrm{i}}-\mathrm{R}\right)$,

where $p i$ is the proportion of the corrected absorbance value of each well to the sum of absorbance values of all wells and $\mathrm{n}$ is the total number of carbon sources (Jay L. Garland, 1997). The Simpson diversity index (D) was determined via the following equations:

$\mathrm{D}=1-[?] \mathrm{P}_{\mathrm{i}}{ }^{2}$.

McIntosh diversity (U):

$\mathrm{U}=, \mathrm{ni}=\mathrm{Ci}-\mathrm{R}$.

\subsection{Principal components analysis}

Prior to principal components analysis (PCA), all absorbance data were converted into Rsi values. The ratio of the AWCD value per samples and the maximum AWCD value in all samples is regarded the relative ratio of six kinds of carbon sources. The ratio of the AWCD value of the sole carbon source and the AWCD of the 31 carbon source is relative for each carbon source, and make a comparison matrix, which could indicated that the use regularity of strong and weak in sole carbon source.

\subsection{Determination of soil properties and enzymatic activities}


Prior to soil physical and chemical analyses, all samples were air-dried at room temperature and passed through a 2-mm soil sieve. Soil $\mathrm{pH}$ was measured with combined electrodes in a 1:2.5 soil to water suspension; available nitrogen was estimated via the alkaline $\mathrm{KMNO}_{4}$ method (Prasad, 1965). Soil organic matter was determined as described previously (Janzen, Bole, Biederbeck, \& Slinkard, 1990).

The activities of urease, peroxidase, and phenol oxidase were determined as described in Saiyacork (Saiyacork, Sinsabaugh, \& Zak, 2002). Catalase activity was measured following the method of Dai et al. (Dai et al., 2017).

\subsection{Statistical analysis}

Statistical differences were tested using two-way analysis of variance (ANOVA), following the generalized linear model procedure of SPSS version 12.0.1 for windows. Fisher's least significant difference (LSD) test was used for mean separation when the analysis of variance showed statistically significant differences $(P<$ $0.05)$.

\section{Results}

\subsection{Soil physico-chemical properties}

The rhizosphere soils from the eight different treatments differed significantly in terms of physico-chemical characteristics (Table 1). Mulberry rhizosphere soils showed a higher $\mathrm{pH}$ value than alfalfa rhizosphere soils; $\mathrm{pH}$ was lower in the intercropped system compared to the monocultures $(P<0.01)$. Nitrogen application resulted in increased $\mathrm{pH}$ values in the monoculture system, but lower $\mathrm{pH}$ values in the intercropping system. Soil organic matter $(\mathrm{OM})$ contents were significantly decreased in mulberry and alfalfa rhizosphere soil with added nitrogen, while in intercropping systems, this trend was alleviated. In the mulberry treatments, OM contents were significantly higher than in the corresponding treatments of alfalfa, except for the monoculture system without nitrogen addition. Available nitrogen (AN) contents were significantly higher in alfalfa rhizosphere soil than in mulberry soil, and the application of nitrogen increased AN values in both systems (monoculture and intercropping). The soil water content (SWC) was considerably higher in mulberry rhizosphere soil than in alfalfa soil. Nitrogen application and intercropping resulted in increased SWC in mulberry soil, but in decreased values in alfalfa soil.

\subsection{Soil enzymatic activities}

Intercropping and nitrogen application increased the activity of urease (SUR) (Table 2). Compared to MN0, SUR activities increased by $28.32 \%(P<0.05), 29.13 \%(P<0.05)$, and $119.47 \%(P<0.01)$ in MNE, M0, and ME, respectively, while, SUR activities in ANE, A0, and AE increased by $2.31 \%(P<0.05), 3.11 \%(P<$ $0.05)$, and $23.25 \%(P<0.05)$, respectively. The SUR activities were higher in mulberry than in alfalfa soil. Nitrogen application increased peroxidase (POD) activity in mulberry soil, while POD activity was decreased in alfalfa soil. The activities of catalase (CAT) were lower in intercropping systems and in mulberry soil with nitrogen application, but there were no significant differences between the alfalfa treatments.

\subsection{Average well color development}

During cultivation, the increase rate of AWCD slowed down after 5 days (Fig. 2); AWCD peaked on day 8 and was stable afterwards. Among the eight treatments, ANE showed the highest metabolic rate; the AWCD of ANE increased from 0 to around 1.37 after 8 days. Treatment MN0 showed the lowest metabolic rate, and AWCD increased to around 1.13, indicating a less efficient carbon substrate use. This shows that incubation time has an effect on microbial activity in the examined systems. In the stable period, there were significant differences in the AWCD among the microbial communities from alfalfa treatments $(P<$ 0.01 ), following the order $\mathrm{ANE}>\mathrm{AE}>\mathrm{ANO}>\mathrm{A} 0$, suggesting that with increasing incubation time, the metabolic activity of the alfalfa microbial community also increases. We observed significant differences in AWCD among the microbial communities of the mulberry treatments $(P<0.01)$, following the order ME $>\mathrm{M} 0>\mathrm{MNE}>\mathrm{MN0}$. The AWCD in the treatments ME, M0 and MNE was increased by $23.8 \%(P<$ $0.01), 21.5 \%(P<0.01)$, and $19.04 \%(P<0.01)$, respectively, compared to MN0. 


\subsection{Metabolic functional diversity indices}

Table 3 shows the mean-AWCD, Shannon diversity index (H'), Simpson diversity index (D), and McINtosh diversity index $(\mathrm{U})$ of the eight treatments. Duncan's multiple range test was separately used in these indices. The mean-AWCD, H', D and U differed significantly $(P<0.05)$ between intercropping systems and treatments with nitrogen application $(P<0.05)$. Intercropping and nitrogen application increased AWCD, H', D, and U of mulberry treatments, with the order ME > M0 [?] MNE > MN0. There were no significant differences between M0 and MNE in the above parameters, indicating that microbial abundance, species richness, and the most common species were similar in M0 and MNE. In alfalfa treatments, intercropping and nitrogen application decreased mean-AWCD and $\mathrm{U}$, following the order AN0 $>\mathrm{ANE}>\mathrm{A} 0>\mathrm{AE}$. There were no significant differences between AN0 and ANE; AE and A0 in terms of H' and D.

\subsection{Principal component analysis (PCA) of carbon source metabolization}

The differences in metabolic characteristics of the intercropping system were directly reflected by the position of the points in the principal vector space. As shown in Figure 3, the multivariate vectors were transformed into two uncorrelated principal component vectors; PC1 accounted for $55.64 \%$ of the total variability and PC2 for $13.24 \%$. For the intercropping system, the point positions of MN0, MNE, M0, ME, AN0, ANE, AE, and A0 were significantly different.

The sample distribution along the $\mathrm{PC}$ axis was relevant to the carbon source use. Based on the results of the PCA, 17 carbon sources constituted PC1, including six amino acids, three carbohydrates, three carboxylic acids, two polymers, two miscellaneous substances, and one amide. Only four carbon sources accounted for PC2, including two carboxyl acids, one polymer, and one carbohydrate. The carbon sources used by mulberry microbes were greatly related to PC1, including amino acids, carbohydrates, and carboxylic acid, while the alfalfa community mainly used carbon sources related to PC2.

\subsection{Six types of carbon sources}

There were significant differences in the use ratios of the six types of carbon source between mulberry and alfalfa treatments (Fig. 4). The relative use rates of carbohydrates, polymers, and miscellaneous substances were higher in mulberry than in alfalfa treatments. However, the relative use rates of carboxylic acids and amino acids were higher in alfalfa treatments. The use ratios of carbohydrates (30.79-37.51\%), polymers (21.14-23.96\%), and miscellaneous substrates (15.32-18.98\%) in mulberry treatments were higher than in the corresponding alfalfa treatments, namely carbohydrates (28.06-31.27\%), polymers (13.94-14.89\%), and miscellaneous substrates (6.20-7.30\%), respectively. The use ratios of carboxylic acids (19.29-20.24\%), amino acids (23.24-26.38\%), and amines/amides (4.17-5.32\%) in alfalfa treatments were higher than those in mulberry treatments, namely acids (11.43-14.97\%), amino acids (7.09-8.37\%), and amines/amides (3.11-5.77\%). These results indicate that mulberry and alfalfa have strategically and complementarily used the six carbon sources. Nitrogen application and intercropping decreased the use ratio of carbohydrates in mulberry treatments, but increased it in alfalfa treatments. The use ratios of amino acids in alfalfa intercropped with mulberry were lower than those in monoculture alfalfa systems, irrespective of nitrogen application. However, nitrogen application decreased the use ratios of amino acids in mulberry, irrespective of the cropping system. The use ratios of polymers in the intercropping of mulberry and alfalfa were higher than those in the monoculture; for mulberry, this was irrespective of nitrogen application. The use ratios of carboxylic acids were higher in mulberry with nitrogen application, without any significant differences between AE, ANE, and AN0. The utilization ratios of amine/amides in alfalfa treatments with nitrogen were lower than those without nitrogen, irrespective of the cropping system.

Among all treatments, the relative use rates of 31 carbon sources differed significantly (Fig. 5). Specifically, the number of carbon sources of the relative use rates exceed $4 \%$ was up to 17 in ME, 15 in M0, 13 in MNE, 9 in MNO, 12 in AE, 7 in A0, 21 in ANE, and 19 in AN0; 2-hydroxy benzoic acid and $\alpha$-D-lactose were scarcely used in all treatments. In addition, $\gamma$-hydroxybutyric acid, L-threonine, and $\alpha$-ketobutyric acid were scarcely use in mulberry treatments (ME, M0, MNE, MN0) and in alfalfa intercropped with mulberry treatments (AE and A0). The relative use rates of D-malic acid, phenylethyl-amine, and glycyl-L- 
glutamic acid in all treatments did not exceed $4 \%$. On the contrary, the relative use rates of L-asparagine, D-glucosamine acid, L-serine, D-mannito, D-cellobiose, N-acetyl-D-glucose, and mehtyl-D-glucoside (except for A0) exceeded $4 \%$ in all treatments. In addition, the relative use rate of D-glucosamine acid in alfalfa exceeded 4\%, while those of Tween 40 and Methyl-D-glucoside exceeded $4 \%$ in all treatments except for A0. Nitrogen application and intercropping increased the relative use rates of L-arginine and $\alpha$-cyclodextrin, which exceeded $4 \%$ in treatments of mulberry (ME, M0, MNE), while the relative use rate of L-arginine in treatments of alfalfa exceeded $4 \%$ except for A0. For $\alpha$-cyclodextrin and itaconic acid, the rates exceeded $4 \%$ in the treatments $\mathrm{AE}$ and $\mathrm{ANE}$, while for putrescine and D-galactonic-acid-lactone, they exceeded $4 \%$ in treatments of mulberry with nitrogen application (MNE and ME). The relative use rates of D-glucosaminic acid, glucose-l-phosphate, and Tween 80 exceeded $4 \%$ in treatments of intercropped mulberry (M0 and ME), while for pyruvic acid methyl ester and D-xylose, the rates exceeded $4 \%$ in intercropped alfalfa with nitrogen application. The relative use rates of 4-hydroxy benzoic acid, putrescine, D-galactonic acid lactone, glucose1-phosphate, Tween 80, and glycogen exceeded $4 \%$ in AN0 and ANE, but were below $4 \%$ in intercropping systems with mulberry ( $\mathrm{AE}$ and $\mathrm{A} 0$ ).

\subsection{Redundancy discriminatory analysis}

The CANOCO software was used to analyze the data in relation to carbon sources and environmental factors. The maximum gradient length in the four axes was 0.464 , with a value below 3 . Therefore, linear model analysis (RDA) was selected. The eigenvalues of the first two ordination axes (carbon source diversity an environmental factors) were 0.573 and 0.213 (Table 4), while the correlation coefficients of the two ordination axes were 1 . The first two ordination eigenvalues accounted for $99.8 \%$ of the total eigenvalues. The correlation coefficients between the first two ranking axes and environmental factors were extremely high, accounting for $99.9 \%$ of the total variance. The axes RDA1 and RDA2 explained 46.4 and $34.9 \%$ of the variation among intercropping system communities, respectively (Table 5).

The greatest differences between treatment communities were observed when comparing mulberry and alfalfa and related to differences in $\mathrm{pH}, \mathrm{OM}, \mathrm{SWC}$ and $\mathrm{AN}$, according to axis length and angle. The second greatest differences were observed between ANE and AN0 and were due to the differences in the activities of PPO, CAT, and POD (Fig. 6). Moreover, POD, CAT, and OPP were positively related to A0 and AN0, while $\mathrm{pH}, \mathrm{AN}$, and SWC were negatively related to AE and ANE. The third greatest differences were observed between ME and MNO, and between MNE and M0, which were related to differences in SWC, AN, SUR, POD, pH, and OM, respectively. The parameters SUR, AN, and SWC were positively related with M0, while $\mathrm{pH}, \mathrm{OM}$, and SWC were positively related to M0 and MNE.

\section{Discussion}

Mulberry leaves and young stems have high protein contents and are rich in numerous active ingredients (Sanchez, 1999). Mulberry can be used as a high-quality feed source for livestock and poultry (Kitahara, Shibata, \& Nishida, 2002) and is characterized by a rapid growth, high yield, and strong resistance, enabling it to adapt to various regions (MACHII, Koyama, \& Yamanouchi, 2001). Previous research has demonstrated that mulberry intercropped with alfalfa increased bacterial richness and diversity (Zhang et al., 2018). For example, Li et al. ( $\mathrm{Li}, \mathrm{Wu}, \&$ Chen, 2007) found that intercropping and intercropping with nitrogen promoted available nutrient enrichment in mulberry rhizosphere soil. In this experiment, intercropping with nitrogen increased available $\mathrm{N}(\mathrm{AN})$ contents both in mulberry and alfalfa treatments, while AN contents were significantly higher in alfalfa than in mulberry treatments. Alfalfa has been described as a strong competitor for available N (Tomm, Walley, Kessel, \& Slinkard, 1995). However, AN contents were significantly decreased in intercropping systems without nitrogen application, mainly because of the relatively small photosynthetically active area of alfalfa (Savoie, Pouliot, \& Sokhansanj, 1995), limiting biological nitrogen fixation after mowing in mid-July. However, mowed alfalfa also absorbs AN from soils and therefore does not act as an $\mathrm{N}$ source or sink (Tomm et al., 1995). In this experiment, intercropping increased the OM contents in mulberry rhizosphere soil, but decreased OM in alfalfa rhizosphere soil. Nitrogen application decreased the OM contents in mulberry and alfalfa rhizosphere soils. Fog et al. (Fog, 1988) have reported that nitrogen application decreased the OM contents in soil. Motavalli et al. (Motavalli, Palm, Parton, 
Elliott, \& Frey, 1995) found that soil organic matter turnover is affected by soil acidity, and previous studies have found that the cultivation of alfalfa can increase soil pH (Su \& Evans, 1996). In our experiment, nitrogen application increased the $\mathrm{pH}$ value both in rhizosphere soil of mulberry and alfalfa, while intercropping resulted in a decreased $\mathrm{pH}$, especially when in combination with nitrogen. This indicates that intercropping could stimulate acid metabolic substances both in alfalfa and mulberry cultivation, which is in agreement with Latati (Latati et al., 2014), who stated that intercropping improved organic acid amounts in the rhizosphere, along with soil phosphorus availability. These acid substances were the main substances for osmotic pressure adjustment, resulting in higher soil water contents (SWC) in intercropping than in monoculture systems. Enzymes catalyze all biochemical reactions and are an integral part of nutrient cycling in the soil (Bandick \& Dick, 1999). Urease catalyzes urea conversion in the soil and therefore has a significant effect on urea use (Delgado-Baquerizo, Grinyer, Reich, \& Singh, 2016). Therefore, the activity of urease directly affects the use ratio of urea. Intercropping and nitrogen application (urea) increased urease activity both in mulberry and alfalfa. However, the activity of polyphenoloxidase decreased in these treatments. The high activity of polyphenoloxidase hinder the further synthesis of humus by the intermediate products of phenol, produced via the mineralization of organic matter, causing the accumulation of phenolic compounds and, consequently, poisoning. Nitrogen application increased the activity of POD in mulberry treatments, but decreased it in alfalfa treatments with nitrogen application and intercropping. The activities of the main enzymes were lower in alfalfa treatments with nitrogen and intercropping, especially in intercropping systems with nitrogen application.

Among the 31 carbon sources on the Biolog-ECO plates, 9 were root exudates. The capability of microorganisms to use different carbon sources was measured by average well color development (AWCD )(J. L. Garland \& Mills, 1991). Samples with larger AWCD values have a higher carbon source use capability and tend to have a higher microbial abundance (Jay L. Garland, 1997). Previous research has indicated that intercropping enhances the diversity of the mulberry rhizosphere soil microbial community and changed the main carbon source types of alfalfa (Zhang et al., 2015). In our study, nitrogen application increased the AWCD values of mulberry intercropped with alfalfa systems. While, intercropped with mulberry and nitrogen application decreased the AWCD values of alfalfa treatments. The changed trends of the diversity indices between mulberry and alfalfa were in accordance with the AWCD values. The Shannon diversity index $(\mathrm{H})$ is greatly influenced by species richness (Y. H. Sun, Yang, Zhao, \& Li, 2012), and higher values indicate a higher metabolic functional diversity (Strong, 2016). The Simpson index (D) is greatly reflected by most common species because it gives more weight to common or dominant species (Simpson, 1949). Interestingly, there were no significant differences of mean AWCD and H and D between monoculture mulberry treatments with nitrogen application (MNE) and intercropped with alfalfa treatments without nitrogen (M0), indicating they use similar carbon sources. The results of the PCA showed that the treatments MNE and M0 were similar in terms of the soil microbial community diversity, showing clusters in using the main carbon source closely related the two principal components in the scattered plots. The relative use rates of six types of carbon sources were significantly different between treatments of mulberry and alfalfa, indicating that these species strategically and complementarily use these carbon sources. According to previous studies, this is mainly related to the advantages of intercropping systems in terms of nitrogen use (Chalk, 1998; Willey, 1979). Compared to monoculture systems, intercropping systems show an improved use of available resources, resulting in higher yields (Hauggaardnielsen \& Jensen, 2001). Cultivars suitable for intercropping should enhance the complementary effects between species (Davis \& Woolley, 1993). In this study, we confirmed that mulberry and alfalfa are suitable for intercropping systems, complementarily using various carbon sources. However, the relative use rates of 2 -hydroxybenzoic acid, $\alpha$-D-lactose, $\gamma$-hydroxybutyric acid, L-threonine, and $\alpha$-ketobutyric acid were less than $2 \%$ in mulberry, while the relative use rates of 2-hydroxybenzoic acid and $\alpha$-D-lactose were less than $2 \%$ in alfalfa treatments. In previous studies, these carbon sources were hardly degraded in plant rhizosphere soil (Deveryshetty, Suvekbala, Varadamshetty, \& Phale, 2010; Wu \& Wang, 2007). Nitrogen application and intercropping increased the numbers of carbon sources with use rates of more than $4 \%$ in mulberry treatments, while intercropping decreased these numbers in alfalfa treatments. This indicates that intercropping and nitrogen application created a more favorable environment for certain microbial groups in mulberry rhizosphere soil (Garau, Silvetti, Deiana, Deiana, \& 
Castaldi, 2011). Redundancy analysis showed that certain soil environmental factors greatly influence the microbial function of intercropped mulberry/alfalfa treatments. Soil physico-chemical parameters were positively related with mulberry treatments, in particular in intercropped mulberry treatments with nitrogen application, while in alfalfa treatments with nitrogen application, we observed a negative correlation. This indicates that nitrogen application and intercropping result in lower carbon metabolic activities of alfalfa soil microbial communities, which is in accordance with the results of Zhao et al. (Zhao, Zeng, He, Chen, \& Wang, 2015). Available nitrogen contents and soil water contents were negatively related with treatments of alfalfa with nitrogen application. However, further studies are needed to evaluate the long-term effects of nitrogen application and intercropping on the functionality of soil microbial communities in mulberry and alfalfa intercropping systems.

\section{Conclusions}

Mulberry and alfalfa showed a strategic complementarity in the use of six types of carbon sources. Intercropping and nitrogen application changed the $\mathrm{pH}$ value and the soil water content both in mulberry and alfalfa rhizosphere soil and resulted in changes in the microbial community and in microbial growth, leading to different carbon use patterns and capability. There is mostly similar in rhizoshere soil microbial communities structure and function between mulberry intercropped with alfalfa without nitrogen application and mulberry monoculture with nitrogen application.

\section{Author contribution}

Xiuli Zhang and Guangyu Sun designe the experiments, analysed data and interpreted the results. Zhiyuan Teng, Huihui Zhang, Dunjiang Cai and Jingyun Zhang executed the experiments. Xiuli Zhang wrote the first draft of the manuscript. All authors revised and edited the drafts of the manuscript and approved its final version.

\section{Funding}

This work was financially supported by the Heilongjiang Province Science Foundation for Youths (Grant No. QC2016018), the National Natural Science Foundation of China (Grant No. 31600508), the Application Technology Research and Development Projects of Heilongjiang Province (Grant No. WB13B104) and the Science and Technology Project of Heilong jiang Farms \& Land Reclamation Administration (Grant No. HNK135-01-056). The authors also declare no conflict of interest. The content of this article is the exclusive responsibility of the authors.

\section{Data Accessibilty Statement}

The data that support the findings of this study are available from the corresponding authors upon reasonable request.

\section{References}

Amador, J. A., \& Görres, J. H. (2007). Microbiological characterization of the structures built by earthworms and ants in an agricultural field.Soil Biology 8 Biochemistry, 39 (8), 2070-2077.

Bandick, A. K., \& Dick, R. P. (1999). Field management effects on soil enzyme activities. Soil Biology $\mathscr{B}$ Biochemistry, 31 (11), 1471-1479.

Baslam, M., Antolín, M. C., Gogorcena, Y., Muñoz, F., \& Goicoechea, N. (2014). Changes in alfalfa forage quality and stem carbohydrates induced by arbuscular mycorrhizal fungi and elevated atmospheric CO2.Annals of Applied Biology, 164 (2), 190-199.

Chalk, P. M. (1998). Dynamics of biologically fixed N in legume-cereal rotations: a review. Australian Journal of Agricultural Research, 49 (3), 303-316.

Chu, H., \& Grogan, P. (2010). Soil microbial biomass, nutrient availability and nitrogen mineralization potential among vegetation-types in a low arctic tundra landscape. Plant $\mathcal{E} 3$ Soil, 329 (1/2), 411-420. 
Classen, A. T., Boyle, S. I., Haskins, K. E., Overby, S. T., \& Hart, S. C. (2003). Community-level physiological profiles of bacteria and fungi: plate type and incubation temperature influences on contrasting soils.Fems Microbiology Ecology, 44 (3), 319-328.

Condron, L., Stark, C., O'Callaghan, M., Clinton, P., \& Huang, Z. (2010). The Role of Microbial Communities in the Formation and Decomposition of Soil Organic Matter : Springer Netherlands.

Dai, W., Ke, X., Li, Z., Gao, M., Wu, L., Chiristie, P., \& Luo, Y. (2017). Antioxidant enzyme activities of Folsomia candida and avoidance of soil metal contamination. Environ Sci Pollut Res Int, 25 (3).

Davis, J. H. C., \& Woolley, J. N. (1993). Genotypic requirement for intercropping. Field Crops Research, 34 (s 3-4), 407-430.

Delgado-Baquerizo, M., Grinyer, J., Reich, P. B., \& Singh, B. K. (2016). Relative importance of soil properties and microbial community for soil functionality: insights from a microbial swap experiment.Functional Ecology, 30 (11), 1862-1873.

Deveryshetty, J., Suvekbala, V., Varadamshetty, G., \& Phale, P. S. (2010). Metabolism of 2-, 3- and 4hydroxybenzoates by soil isolates Alcaligenes sp. strain PPH and Pseudomonas sp. strain PPD. Fems Microbiology Letters, 268 (1), 59-66.

Dong, X., Yao, H. Y., De-Yong, G. E., \& Huang, C. Y. (2008). Soil microbial community structure in diverse land use systems:A comparative study using Biolog,DGGE,and PLFA analyses. Pedosphere, 18 (5), 653-663.

Fog, K. (1988). The effect of added nitrogen on the rate of decomposition of organic matter. Biological Reviews, 63 (3), 433-462.

Garau, G., Silvetti, M., Deiana, S., Deiana, P., \& Castaldi, P. (2011). Long-term influence of red mud on As mobility and soil physico-chemical and microbial parameters in a polluted sub-acidic soil. Journal of Hazardous Materials, 185 (2), 1241-1248.

Garland, J. L. (1997). Analysis and interpretation of community-level physiological profiles in microbial ecology. Fems Microbiology Ecology, 24 (4), 289-300.

Garland, J. L., \& Mills, A. L. (1991). Classification and characterization of heterotrophic microbial communities on the basis of patterns of community-level sole-carbon-source utilization. Appl Environ Microbiol, 57 (8), 2351-2359.

Giller, K. E., Witter, E., \& Mcgrath, S. P. (1998). Toxicity of heavy metals to microorganisms and microbial processes in agricultural soils: a review. Soil Biology \& Biochemistry, 30 (10-11), 1389-1414.

Hauggaardnielsen, H., \& Jensen, E. S. (2001). Evaluating pea and barley cultivars for complementarity in intercropping at different levels of soil N availability. Field Crops Research, 72 (3), 185-196.

Heichel, G. H., \& Vance, C. P. (1979). Nitrate-N and Rhizobium Strain Roles in Alfalfa Seedling Nodulation and Growth 1. Crop Science, 19 (4), 512-518.

Huang, N., Wang, W., Yao, Y., Zhu, F., Wang, W., \& Chang, X. (2017). The influence of different concentrations of bio-organic fertilizer on cucumber Fusarium wilt and soil microflora alterations. Plos One, 12 (2), e0171490.

Janzen, H. H., Bole, J. B., Biederbeck, V. O., \& Slinkard, A. E. (1990). Fate of N applied as green manure or ammonium fertilizer to soil subsequently cropped with spring wheat at three sites in western Canada.Canadian Journal of Soil Science, 70 (3), 313-323.

Jiao. (2006). Effects of continuous cucumber cropping and alternative rotations under protected cultivation on soil microbial community diversity. Plant \&3 Soil, 284 (1-2), 195-203. 
Kitahara, N., Shibata, S., \& Nishida, T. (2002). Management and utilization of mulberry for forage in Japan. 1. Productivity of the mulberry-pasture association system and nutritive value of mulberry.Physical Chemistry Chemical Physics Pccp, 15 (23), 9335-9342.

Ladygina, N., \& Hedlund, K. (2010). Plant species influence microbial diversity and carbon allocation in the rhizosphere. Soil Biology $\mathscr{E}$ Biochemistry, 42 (2), 162-168.

Latati, M., Blavet, D., Alkama, N., Laouf, H., Drevon, J. J., Gerard, F., . . . Ounane, S. M. (2014). The intercropping cowpea-maize improves soil phosphorus availability and maize yields in an alkaline soil.Plant Es Soil, 385 (1-2), 1-11.

Li, Z., Wu, X., \& Chen, B. (2007). Changes in transformation of soil organic C and functional diversity of soil microbial community under different land uses. Agricultural Sciences in China, 6 (10), 1235-1245.

Lipman, J. G. (1912). associative growth of legumes and non-legumes.

Liu, Y. R., Delgado-Baquerizo, M., Wang, J. T., Hu, H. W., Yang, Z., \& He, J. Z. (2018). New insights into the role of microbial community composition in driving soil respiration rates. Soil Biology 8 Biochemistry, $118,35-41$.

MACHII, Koyama, A., \& Yamanouchi, H. (2001). A list of Morphological and Agronomical Traits of Mulberry Genetic Resources. Miscellaneous Publication of the National Institute of Sericultural \& Entomological Science, 18 (6), 433\&ndash;442.

Mao, L., Zhang, L., Li, W., Werf, W. V. D., Sun, J., Spiertz, H., \& Li, L. (2012). Yield advantage and water saving in maize/pea intercrop.Field Crops Research, 138 (3), 11-20.

Motavalli, P. P., Palm, C. A., Parton, W. J., Elliott, E. T., \& Frey, S. D. (1995). Soil pH and organic C dynamics in tropical forest soils: Evidence from laboratory and simulation studies. Soil Biology E Biochemistry, 27 (12), 1589-1599.

Prasad, R. (1965). Determination of Potentially Available Nitrogen in Soils - A Rapid Procedure. Plant $\&$ Soil, 23 (2), 261-264.

Saiyacork, K. R., Sinsabaugh, R. L., \& Zak, D. R. (2002). The effects of long term nitrogen deposition on extracellular enzyme activity in an Acer saccharum forest soil. Soil Biology 83 Biochemistry, 34 (9), 1309-1315.

Sanchez, M. D. (1999). Mulberry: an exceptional forage available almost worldwide. World Review of Animal Production, 93 (93), 36-46.

Savoie, P., Pouliot, M., \& Sokhansanj, S. (1995). Potential impact of mowing-maceration on an alfalfa dehydration plant. Canadian Society for Bioengineering .

Simpson, E. H. (1949). The Measurement of Diversity. Nature, 163 (4148), 688.

Stefanowicz, A. (2006). The Biolog Plates Technique as a Tool in Ecological Studies of Microbial Communities. Polish Journal of Environmental Studies, 15 (5), 669-676.

Strong, W. L. (2016). Biased richness and evenness relationships within Shannon-Wiener index values. Ecological Indicators, 67, 703-713.

$\mathrm{Su}, \mathrm{C}$., \& Evans, L. J. (1996). Soil solution chemistry and alfalfa response to CaCO3 and MgCO3 on an acidic Gleysol. Canadian Journal of Soil Science, 76 (1), 41-47.

Sun, Y. H., Yang, Z. H., Zhao, J. J., \& Li, Q. (2012). Functional Diversity of Microbial Communities in Sludge-Amended Soils.Physics Procedia, 33 (6), 726-731.

Sun, Y. M., Zhang, N. N., Wang, E. T., Yuan, H. L., Yang, J. S., \& Chen, W. X. (2009). Influence of intercropping and intercropping plus rhizobial inoculation on microbial activity and community composition 
in rhizosphere of alfalfa (Medicago sativa L.) and Siberian wild rye (Elymus sibiricus L.). Fems Microbiology Ecology, 70 (2), 62-70.

Tang, X., Bernard, L., Brauman, A., Daufresne, T., Deleporte, P., Desclaux, D., . . . Hinsinger, P. (2014). Increase in microbial biomass and phosphorus availability in the rhizosphere of intercropped cereal and legumes under field conditions. Soil Biology \& Biochemistry, 75 , 86-93.

Teuber, L. R., Levin, R. P., Sweeney, T. C., \& Phillips, D. A. (1984). Selection for N Concentration and Forage Yield in Alfalfa. Crop Science, 24 (3), 553-558.

Tomm, G. O., Walley, F. L., Kessel, C. V., \& Slinkard, A. E. (1995). Nitrogen Cycling in an Alfalfa and Bromegrass Sward via Litterfall and Harvest Losses. Agronomy Journal, 87 (6), 1078-1085.

Wang, W. X., Yang, H. J., Bo, Y. K., Ding, S., \& Cao, B. H. (2012). Nutrient composition, polyphenolic contents, and in situ protein degradation kinetics of leaves from three mulberry species.Livestock Science, $146(2-3), 203-206$.

Willey, R. W. (1979). Intercropping-Its Importance and Research Needs : Part 1. Competition and Yield Advantages. Field Crop Abstracts, 32 .

Wu, F., \& Wang, X. (2007). Effect of monocropping and rotation on soil microbial community diversity and cucumber yield and quality under protected cultivation. Scientia Agricultura Sinica (761), 555-561.

Zhang, M. M., Hong, A. O., Xin, L. I., Zhang, J. Y., Wang, N., Cheng-Mei, J. U., . . . Sun, G. Y. (2015). Effects of Intercropping between Mulberry and Alfalfa on Soil Enzyme Activities and Microbial Community Diversity in Rhizophere. Acta Agrestia Sinica .

Zhang, M. M., Wang, N., Hu, Y. B., \& Sun, G. Y. (2018). Changes in soil physicochemical properties and soil bacterial community in mulberry (Morus alba L.)/alfalfa (Medicago sativa L.) intercropping system.Microbiologyopen, 12 (12), e0189781.

Zhao, J., Zeng, Z., He, X., Chen, H., \& Wang, K. (2015). Effects of monoculture and mixed culture of grass and legume forage species on soil microbial community structure under different levels of nitrogen fertilization. European Journal of Soil Biology, 68 , 61-68.

\section{Hosted file}

Figure 1.docx available at https://authorea.com/users/303255/articles/433374-nitrogenapplication-and-intercropping-advantageously-promote-microbial-community-diversity-andphysio-chemical-characteristics-in-mulberry-and-alfalfa-rhizosphere-soil 